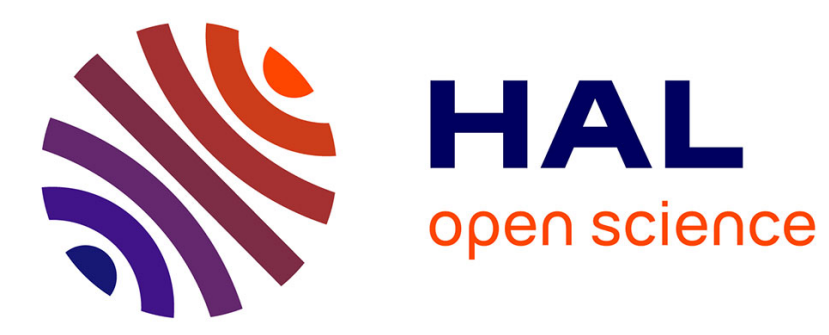

\title{
Mémoire, lieux et invention spatiale dans la peinture italienne des XIIIe et XIVe siècles
}

Jean-Philippe Antoine

\section{To cite this version:}

Jean-Philippe Antoine. Mémoire, lieux et invention spatiale dans la peinture italienne des XIIIe et XIVe siècles. Annales, 1993, 48 (6), pp.1447-1469. halshs-00595020

\section{HAL Id: halshs-00595020 \\ https://shs.hal.science/halshs-00595020}

Submitted on 23 May 2011

HAL is a multi-disciplinary open access archive for the deposit and dissemination of scientific research documents, whether they are published or not. The documents may come from teaching and research institutions in France or abroad, or from public or private research centers.
L'archive ouverte pluridisciplinaire HAL, est destinée au dépôt et à la diffusion de documents scientifiques de niveau recherche, publiés ou non, émanant des établissements d'enseignement et de recherche français ou étrangers, des laboratoires publics ou privés. 


\section{Persée}

http://www.persee.fr

\section{Mémoire, lieux et invention spatiale dans la peinture italienne des XIIle et XIVe siècles}

Jean-Philippe Antoine

Annales, Année 1993, Volume 48, Numéro 6

p. $1447-1469$

Voir l'article en ligne

Memory Places and Spatial Invention in 13th and 14th Century Italian Painting. J.-Ph ANTOINE. This article investigates the history of the art of memory the late Middle Ages and Early Renaissance in Italy and its bearing on the production of material images. After mapping the stages of the rediscovery of antique Roman techniques of images and places it examines the relation of these memory techniques to the transfor mation of pictorial space that takes place in mural painting at the end of the 13th and beginning of the 14th century. The mental habit of locating images into places leads to new awareness of the figurai interaction between architectural and pictorial space in turn responsible for such ensembles as the Higher Church of Assisi or Scro-vegni Chapel in Padua images and architecture are there combined to create region for memory resulting in pictorial plane with new spacious quality and allowing viewers to experience their surroundings as physical extension of mental space. This moment in the history of images is not primitive version of perspectiva artificialis but type of image with its own coherent set of principles explaining both the need for illusionistic space and its limits and accounting for the phantasti- cal quality of space in 14th century Italian painting.

\section{Avertissement}

L'éditeur du site «PERSEE » - le Ministère de la jeunesse, de l'éducation nationale et de la recherche, Direction de l'enseignement supérieur, Sous-direction des bibliothèques et de la documentation - détient la propriété intellectuelle et les droits d'exploitation. A ce titre il est titulaire des droits d'auteur et du droit sui generis du producteur de bases de données sur ce site conformément à la loi n`98-536 du 1er juillet 1998 relative aux bases de données.

Les oeuvres reproduites sur le site «PERSEE » sont protégées par les dispositions générales du Code de la propriété intellectuelle.

Droits et devoirs des utilisateurs

Pour un usage strictement privé, la simple reproduction du contenu de ce site est libre.

Pour un usage scientifique ou pédagogique, à des fins de recherches, d'enseignement ou de communication excluant toute exploitation commerciale, la reproduction et la communication au public du contenu de ce site sont autorisées, sous réserve que celles-ci servent d'illustration, ne soient pas substantielles et ne soient pas expressément limitées (plans ou photographies). La mention Le Ministère de la jeunesse, de l'éducation nationale et de la recherche, Direction de l'enseignement supérieur, Sous-direction des bibliothèques et de la documentation sur chaque reproduction tirée du site est obligatoire ainsi que le nom de la revue et- lorsqu'ils sont indiqués - le nom de l'auteur et la référence du document reproduit.

Toute autre reproduction ou communication au public, intégrale ou substantielle du contenu de ce site, par quelque procédé que ce soit, de l'éditeur original de l'oeuvre, de l'auteur et de ses ayants droit.

La reproduction et l'exploitation des photographies et des plans, y compris à des fins commerciales, doivent être autorisés par l'éditeur du site, Le Ministère de la jeunesse, de l'éducation nationale et de la recherche, Direction de l'enseignement supérieur, Sous-direction des bibliothèques et de la documentation (voir http://www.sup.adc.education.fr/bib/ ). La source et les crédits devront toujours être mentionnés. 


\section{LECTURES DES OEUVRES}

\section{MÉMOIRE, LIEUX ET INVENTION SPATIALE dans la peinture italienne des $\mathrm{XIII}^{\mathrm{e}}$ et $\mathrm{XIV}^{\mathrm{e}}$ siècles}

Jean-Philippe Antoine

La peinture italienne des $\mathrm{XIII}^{\mathrm{e}}$ et $\mathrm{XIV}^{\mathrm{e}}$ siècles possède dans l'histoire de l'art, depuis les Vies de Vasari, un statut exceptionnel : celui d'origine de la «peinture occidentale». En faisant de Cimabue et de Giotto les primi lumi d'une «manière moderne " encore en enfance, Vasari mettait en effet en place un discours qui n'a cessé de nourrir les histoires de l'art successives. La fin du Duecento et le Trecento y représentent, après le sombre Moyen Age, le début d'une «renaissance » des arts inspirée de l'Antiquité. Mais ce commencement ne se connaît pas pour tel, et sa vérité est le fruit d'un accomplissement ultérieur: l'invention au Quattrocento de la perspective, et les développements qu'en proposent Michel-Ange, Léonard de Vinci et Raphaël. D'où la double caractérisation dont la peinture des XIII et $\mathrm{XIV}^{\mathrm{e}}$ siècles a fait, alternativement ou parfois simultanément, l'objet : œuvre de "primitifs ", qu'identifie leur incapacité à mettre en pratique sans erreur les critères spatiaux définis à partir $d u X^{e}$ siècle, ou au contraire, pour citer le titre de l'ouvrage fameux d'Erwin Panofsky, œuvre d' «avant-courriers » de la Renaissance, auteurs valeureux d' " une première rupture par rapport aux principes médiévaux de représentation du monde visible au moyen du trait et de la couleur $»^{1}$.

Dans l'un et l'autre cas, ce qui garantit le statut d'origine de cette peinture est son analyse à partir de l'élaboration nouvelle de l'espace pictural réalisée par Brunelleschi et formulée par Alberti et ses successeurs. Celle-ci, bientôt connue sous le nom de perspectiva artificialis, a pour objet ce que E. Panofsky désignera par «espace du tableau », un tableau explicitement

* L'auteur et l'éditeur remercient la Bibliothèque Apostolique Vaticane qui a bien voulu autoriser la reproduction des documents lui appartenant.

1. E. Panofsky, La Renaissance et ses avant-courriers dans l'art d'Occident, Paris, 1976, Stockholm, 1960, p. 37. 


\section{LECTURES DES GUVRES}

comparé à « une fenêtre par laquelle nous regardons une portion du monde visible $»^{2}$.

Cette construction historique, dont ce n'est pas ici le lieu d'explorer en détail les enjeux ${ }^{3}$, pèche de double façon. Elle conduit à appliquer à des œuvres qui apparaissent dès le dernier quart du XIII ${ }^{\mathrm{e}}$ siècle des catégories élaborées quelque cent cinquante ans plus tard. Elle est donc au sens strict anachronique. Plus gravement, elle interdit l'accès aux questions qui se posaient pour les fabricants d'images des Duecento et Trecento, questions qui, pour être demeurées longtemps occultées, n'en sont pas moins réelles.

Il existe en effet tout un pan de la culture médiévale et renaissante qui permet d'articuler en des termes contemporains la question de l'image et de son espace: l'art de la mémoire, ou encore la memoria, c'est-à-dire l'ensemble des prescriptions et des pratiques mnémoniques inspirées de l'Antiquité qui gouvernent l'éducation, la prédication et de façon générale la formalisation des savoirs. Après avoir été négligé, sinon nié, pendant plusieurs siècles, l'art de la mémoire a depuis une trentaine d'années resurgi à la lumière, d'abord grâce aux travaux pionniers de Frances Yates ${ }^{4}$ et Paolo Rossi $^{5}$, puis avec la récente somme de Mary Carruthers ${ }^{6}$.

Dans une large mesure, la memoria fait usage de constructions d'images. La place croissante qu'elle occupe dans la société de la fin du Moyen Age, ainsi que les formes nouvelles qu'elle y prend, permettent d'articuler de façon satisfaisante non seulement le rôle éminent accordé en général à l'image par cette société - ce que Huizinga appelait déjà la "cristallisation sur les images »-, mais aussi, et plus précisément, les développements proprement picturaux qui l'accompagnent ${ }^{7}$.

Avant de poursuivre, un mot d'avertissement est nécessaire. Comme on le verra bientôt, les constructions d'images de la memoria mettent en jeu un double mécanisme : la production, le plus souvent à partir du registre verbal, d'une image de l'objet-souvenir; son placement dans une série de lieux. C'est à ce second aspect, et à ses conséquences sur l'invention spatiale de la peinture, que l'on s'intéressera dans les pages qui suivent, à l'exclusion volontaire du premier ${ }^{8}$.

2. Ibidem, p. 128. Le titre de l'ouvrage remarquable consacré par John White à la transformation de l'espace pictural dans la peinture italienne du XII ${ }^{\mathrm{e}}$ au XV $\mathrm{XV}^{\mathrm{e}}$ siècle, The Birth and Rebirth of Pictorial Space, Londres, 1957, résume à lui seul l'ensemble de cette problématique.

3. Je renvoie pour cela aux travaux de Robert KLEIN, ainsi qu'aux récentes et très pertinentes analyses de Georges Didi-Huberman, Devant l'image, Paris, 1990. Dans un travail inédit, Peindre le nom du souvenir, j'analyse le rôle spécifique que joue dans cette construction l'«idéologie » panofskienne de la perspective.

4. The Art of Memory, Londres, 1966 (trad. fr. 1975).

5. Clavis universalis, Milan-Naples, 1960.

6. The Book of Memory. A Study of Memory in Medieval Culture, Cambridge, 1990. Cf. compte rendu dans ce même numéro. On ajoutera à ces travaux le recueil collectif récemment édité par Lina Bolzoni et Pietro Corsi, La cultura della memoria, Bologne, Il Mulino, 1992.

7. Frances YATES a eu la première l'intuition de cette relation. Cf. le chapitre IV de L'art de la mémoire, «La mémoire médiévale et la formation d'un système d'images », pp. 95-118.

8. Sur la question des «mots dans l'image ", je me permets de renvoyer le lecteur à un travail plus ancien, "Ad perpetuam memoriam. Les nouvelles fonctions de l'image peinte en Italie: 1250-1400 ", Mélanges de l'École française de Rome. Moyen Age-Temps modernes, 100, 1988-2, pp. 541-615, en particulier pp. 560-577, ainsi qu'aux ouvrages cités dans les notes 4 à 6 . 


\section{Les deux mémoires}

Les différentes formes de la memoria médiévale sont tributaires de ses versions antiques. Celles-ci sont accessibles, pour les hommes du Moyen Age comme aujourd'hui pour nous, à travers un petit nombre de textes, essentiellement latins : le De l'invention de Cicéron, l'anonyme Rhétorique à Hérennius, attribuée durant tout le Moyen Age elle aussi à Cicéron, et l'Institution de l'orateur de Quintilien. Ces textes organisent la memoria autour de deux axes principaux et divergents. L'art de la mémoire cicéronien, ou plus exactement hérennien, puisque la Rhétorique en offre la version la plus complète et cohérente, repose sur la fabrication d'un système de lieux et d'images, que son auteur compare aux tablettes de cire qui servaient alors à prendre des notes. Il consiste en l'établissement d'un itinéraire réglé dans une série de lieux architecturaux (maison, palais, colonnade), réels ou imaginaires. Après avoir appris cette série de lieux par cœur, de façon à pouvoir les parcourir mentalement dans tous les sens, on fabrique des images des objets-souvenirs que l'on veut mémoriser, et on les y range dans l'ordre désiré. Lorsque l'on voudra retrouver le souvenir, on parcourra les lieux jusqu'à l'image qui figure le souvenir recherché. Celle-ci déclenche la réminiscence.

La fabrication des lieux comme celle des images est assujettie à des règles. La première met en jeu une série de préceptes concernant leurs caractéristiques pour le regard : les lieux doivent être peu fréquentés, pour ne pas confondre les images qui y sont placées; variés; de taille moyenne; bien éclairés; établis à distance régulière. La seconde repose essentiellement sur un principe de transformation des mots en image par association libre, et sur la nécessité que les images créées soient agentes, c'est-à-dire à la fois frappantes et en action. Ce dernier trait a pour conséquence la construction obligatoire de l'image autour de figures humaines: l'image de mémoire a l'allure d'une scène, au sens à la fois local et théâtral du mot, ou encore, pour reprendre l'expression que Freud applique au rêve, d'une "illusion d'événement"; la mémoire de qui pratique cet art se présente, elle, comme une série de pièces où prennent place des scènes frappantes: violentes, ridicules ou nobles, alternativement très belles ou très laides.

Si Quintilien décrit lui aussi de façon détaillée l'art des lieux et des images, il est loin de lui accorder la même faveur que Cicéron et l'auteur du Ad Herennium. Il lui préfère un ensemble de prescriptions organisées autour de la lecture du livre : diviser le texte en petites unités; fabriquer en marge des marques - images ou mots - désignant le sujet traité ; apprendre toujours sur le même manuscrit, et en retenir les accidents. C'est l'image du texte qui commande alors le processus de mémorisation. En la visualisant mentalement, ainsi que les circonstances qui entourent son apprentissage, on en retrouvera le contenu.

Des deux principes de memoria ici définis, c'est le second qui va dominer la fin de l'Antiquité et les premiers siècles du Moyen Age. L'opposition par- 


\section{LECTURES DES GUVRES}

tielle entre mémoires hérennienne et quintilianienne ${ }^{9}$ est en effet partie prenante du divorce croissant entre la culture figurative hellénistique et romaine dont Cicéron et ses contemporains étaient encore tributaires, et une naissante «culture de l'écrit » dont l'invention du codex, contemporaine de Quintilien, est une autre manifestation ${ }^{10}$. Cette culture de l'écrit et du livre se renforce avec la christianisation progressive de la société antique. Les techniques de mémorisation centrées sur la lecture en sont, comme le codex $^{11}$, l'expression. Dans cette configuration nouvelle du mot et de l'image, la disposition du texte, l'image de sa forme écrite, deviennent les plus précieux auxiliaires de la mémoire. La succession des pages est une série ordonnée de lieux, chacun distingué par l'arrangement de mots qu'il abrite, et par des marques composées, souvent pêle-mêle, de mots et d'images.

La tradition quintilianienne répugne en général à la construction d'imagines agentes, lui préférant, lorsqu'elle fait usage d'images, des signes ou pictogrammes simples ${ }^{12}$. Mais plus que l'absence des images incongrues et frappantes recommandées par la tradition hérennienne, qui est loin d'être partout vérifiée ${ }^{13}$, c'est l'absence de la problématique des lieux architecturaux qui définit ce premier état de la memoria médiévale, les constructions d'images ayant pour lieu principal le livre, c'est-à-dire le support physique du texte. Il faut attendre la fin du $\mathrm{XI}^{\mathrm{e}}$ siècle et le début du $\mathrm{XII}^{\mathrm{e}}$ siècle pour assister à la résurgence d'un intérêt actif pour les préceptes locaux de la Rhétorique à Hérennius, dont témoigne la multiplication des commentaires. Ces préceptes vont être de nouveau analysés et enseignés dans les écoles cathédrales et canoniales, puis bientôt dans les universités naissantes. Surtout, des tentatives sont faites pour adapter de manière contemporaine les préceptes antiques, tentatives dont le témoignage le plus marquant est l'œuvre de Hugues de Saint-Victor, l'un des plus influents penseurs et pédagogues du XII ${ }^{\mathrm{e}}$ siècle.

9. La transmission de la tradition « quintilianienne » ne s'effectuera pas en général au travers de la connaissance directe de l'Institution de l'orateur, mais plutôt par celle des rhéteurs basantiques comme Julius Victor ou Martianus Capella, bientôt relayés par Isidore de Séville.

10. J'emprunte ici aux excellentes remarques d'Agnès Rouveret, dans «Peinture et art de la mémoire : le paysage et l'allégorie dans les tableaux grec et romain ", Comptes rendus de l'Académie des Inscriptions, 1983, pp. 571-588, p. 577. Voir également, du même auteur, Histoire et imaginaire de la peinture ancienne, Rome, 1989, chapitre VI : "Artificiosa memoria. L'invention des images".

11. La métaphore fondamentale de la technique des lieux avait pour l'un de ses termes les tablettes de cire qui sont l'ancêtre direct du codex, et non pas les rouleaux de papyrus, jusque-là seuls vrais «livres ». La cire renvoie par ailleurs à la métaphore platonicienne puis aristotélicienne du travail de la mémoire : l'empreinte d'un sceau, métaphore que reprennent QuiNTILIEN (Institutio, XI, II, 4), et à sa suite la plupart des écrivains médiévaux. Avec l'établissement du codex, le livre investit donc une forme à destination mnémonique. Il y a là comme un courtcircuit de la métaphore lieux/images-tablettes/lettres, réduite à ses seuls seconds termes.

12. Par exemple une ancre pour la navigation, des armes pour la guerre, etc.

13. En témoignent par exemple certains manuscrits des Institutions cassiodoriennes des ${ } \mathrm{X}^{\mathrm{C}}$ et $\mathrm{X}^{\mathrm{e}}$ siècles, ou encore le célèbre psautier d'Utrecht ( $\mathrm{IX}^{\mathrm{c}}$ siècle), les uns et les autres dotés d'images et de scènes reposant sur la figuration de jeux de mots effectués à partir du texte. Dans les deux cas, on a affaire à des œuvres destinées à l'enseignement (le psautier est le premier livre de lecture) et à des étudiants débutants. 


\section{La mémoire victorine}

L'œuvre de Hugues a pour l'histoire de la memoria et des images médiévales une importance capitale. Elle est l'une des seules où le lien entre préceptes théoriques et pratique des images soit établi chez un même auteur. Un premier ensemble est constitué par le Chronicon, résumé de l'histoire universelle rédigé sous forme de tables, et sa préface, le De tribus maximis circumstantiis gestorum, qui en fournit le mode d'emploi. Un second est formé du traité De Arca Noe morali, consacré à l'élaboration des significations allégoriques et morales de l'Arche de Noé, et de son pendant, le $D e$ Arca Noe mystica, qui décrit la fabrication d'une image de celle-ci où loger les premières. Ces deux ensembles représentent symétriquement, le premier, des techniques de base destinées aux commençants, le second un système complexe et bariolé, permettant à un penseur érudit de ranger et d'élaborer l'ensemble des savoirs dont il a l'usage, à savoir une quantité énorme de concepts, de faits et d'énoncés, ainsi que les relations qu'ils entretiennent les uns avec les autres.

Si la préface du Chronicon reprend en grande partie la vulgate " quintilianienne » et désormais classique de la memoria (diviser le texte, mémoriser son image physique ainsi que les circonstances de son apprentissage), l'autre méthode qu'elle met en œuvre - le placement des objets à mémoriser (en l'occurrence les Incipit des Psaumes) sur une ligne numérotée destinée à être visualisée - représente un retour à la logique des lieux, logique déjà implicite dans les premiers paragraphes du texte, où Hugues comparait l'opération de la memoria à celle du changeur classant les monnaies dans les différents compartiments de sa bourse.

C'est cette logique que déploie pleinement la peinture de l'Arche dont le De Arca mystica propose la construction ${ }^{14}$. Cette pictura est la combinaison d'une image de l'Arche, conçue sous la forme d'une pyramide à base rectangulaire formée de trois degrés, et réalisée sous forme de plan, d'une mappamundi ovale qui l'entoure, et d'un Christ en majesté qui enserre le tout. A l'intérieur de cette structure viennent s'insérer des tables chronologiques, des images des vices et des vertus, ainsi que des personnages bibliques et historiques. Il est hors de question d'analyser ici en détail cette construction monumentale, mais son trait principal mérite d'être isolé : la structure de l'image est formée par un plan architectural entouré d'une carte. Dans l'un et l'autre cas, des lieux sont produits et représentés, dans le plan avec une précision géométrique obsessive, dans la carte sous forme d'une topologie plus vague.

Il existait avant le $\mathrm{XII}^{\mathrm{e}}$ siècle une tradition limitée d'usage de diagrammes, qui remonte au moins à Isidore de Séville. Si Hugues ne se fait pas faute de les utiliser, comme quelques-uns de ses contemporains avant lui, il en fait un usage neuf, par la liaison qu'il établit entre diagramme et plan. Les lieux diagrammatiques, qui n'avaient d'autre existence que celle de place mentale, deviennent ainsi dans l'image de l'Arche l'indice de lieux définis

14. Il est important que le traité se présente comme la description de la construction de l'image, et non comme une simple ekphrasis. 


\section{LECTURES DES CEUVRES}

architecturalement. Ils cessent d'indiquer un simple ordre de rangement, pour faire référence à une spatialité extérieure à celle de l'esprit, et, dans le cas de l'Arche, mesurable. La mappamundi qui entoure le plan procède, bien que de manière moins accentuée ${ }^{15}$, de la même logique. En opposition aux diagrammes précédemment en usage, son utilisation dans le contexte de la memoria fait elle aussi signe vers la spatialité des lieux.

Ce mouvement du diagramme vers le plan, et du lieu logique vers le lieu physique, n'est pas l'apanage exclusif de l'œuvre de Hugues. On le retrouve chez son disciple Richard de Saint-Victor, comme à leur suite chez d'autres auteurs cisterciens ou prémontrés. Poursuivant l'explication de la structure des bâtiments bibliques entamée par Hugues, Richard écrit ainsi un traité Sur la vision d'Ezéchiel qu'il consacre presque entièrement à la description topographique et architecturale de la Jérusalem céleste. La destination de celui-ci est double : démontrer la littéralité de la description biblique ; créer avec les constructions décrites une série de lieux où ranger énoncés et faits, sous forme d'images. C'est le sens qu'a aussi le Tabernacle tripartite d'Adam le Prémontré, un traité qui utilise la description du Tabernacle de l'Arche d'Alliance pour y loger des significations morales et allégoriques, et dans lequel cette description devient pour partie celle d'une église contemporaine. A chaque fois, le travail allégorique est rendu possible par la construction préalable d'une série de lieux qui en structurent l'apprentissage et la mémoire. Ces lieux ne sont pas destinés à l'avance à recevoir telle ou telle interprétation ou figure. Ils forment une structure vacante, sur laquelle disposer, selon les nécessités de la tâche poursuivie, les énoncés à mettre en mémoire ${ }^{16}$.

Cette première résurgence de la mémoire hérennienne possède plusieurs traits distinctifs. Tout d'abord, elle est retour aux principes de la Rhétorique antique, plutôt qu'au détail des préceptes qu'elle énonçait. La forme partiellement diagrammatique que prend la disposition des images est originale, ainsi que la présence d'inscriptions écrites dans les images peintes qui servent à matérialiser et ancrer la construction mentale de l'image ${ }^{17}$.

Son second trait est son enracinement dans le renouveau monastique qui s'opère dans le courant du XII ${ }^{\mathrm{e}}$ siècle : les canons victorins ou les cisterciens en sont les premiers artisans, qui donnent pour un temps à la problématique architecturale du lieu son double caractère biblique et ecclésial.

Enfin elle est liée à l'usage d'images matérielles. La pictura de l'Arche hugonienne, par exemple, est une peinture de dimensions monumentales, réalisée soit sur des parchemins cousus ensemble par dizaines, soit sur un

15. L'espace des lieux n'y est pas mesurable, mais seule l'indication de leurs distances et de leurs directions relatives.

16. D'où la croissance symétrique d'écrits présentés sous forme d'index ou de nomenclatures (Quaestiones, Distinctiones, etc.). Ceux-ci forment une matière première que la mise en lieux permet ensuite de composer selon l'ordre requis par le lecteur et réclamé par les circonstances : classe, prêche, sermon universitaire, etc.

17. Le caractère hérennien de cet art de la mémoire ne fait pas de doute, malgré les différences notables qui le distinguent de sa source. Un bon exemple en est fourni par la Parisiana Poetria de Jean de Garlande, qui attribue explicitement à «Tullius ", c'est-à-dire au pseudoCicéron, un système qui reprend dans ses grandes lignes l'art décrit par Hugues de Saint-Victor. 
mur, comme la mappamundi de l'église de Chalavoy-Millon ${ }^{18}$. Cette peinture sert à son tour de lieu à des visualisations qui n'ont d'autre existence que mentale.

La diffusion de cette mémoire "hérenno-victorine " va s'effectuer dans plusieurs directions: des écoles canoniales vers les universités ${ }^{19}$, d'abord parisiennes, puis italiennes ${ }^{20}$; puis, au XIII ${ }^{\mathrm{e}}$ siècle, chez les franciscains et les dominicains, les premiers plus attentifs au caractère pictural de la méthode, les seconds à ses capacités d'ordonnance. Mais si son ancrage monastique reste important tout au long du XIII ${ }^{\mathrm{e}}$ siècle et même du XIV ${ }^{\mathrm{e}}$ siècle, cette forme de memoria déborde maintenant largement la sphère des clercs pour envahir la société civile. Cela est particulièrement vrai de l'Italie, où l'évolution sociale et politique vers l'età comunale, combinée à la présence du passé romain, donne à la rhétorique "cicéronienne », et à sa quatrième partie, la memoria, un statut inégalé dans les autres pays d'Europe.

\section{Mémoire et rhétorique au Duecento}

L'un des meilleurs témoins de l'expansion des techniques de la memoria à la société civile italienne est le juriste et rhétoricien Boncompagno da Signa, actif entre les dernières années du XII ${ }^{\mathrm{e}}$ siècle et 1240 . Dans sa Rhetorica novissima (1235), Boncompagno donne au domaine de la mémoire artificielle une ampleur nouvelle. L'énumération de ses instruments, qui se poursuit durant deux pages, inclut livres, tablettes, images peintes, sculptures et monuments, mais aussi sceaux, supplices, dons et jusqu'aux claques que les évêques administrent lors de l'onction des adultes. Aux écrits et aux images viennent ainsi s'ajouter les signes de la puissance publique, les sons et musiques qui rythment les jours, enseignes et emblèmes, ou encore les instruments d'un état ou d'un métier. Boncompagno définit ainsi un efficace virtuel de la memoria artificialis qui embrasse l'ensemble de l'expérience: ce qu'Albert le Grand appellera, lui aussi dans le contexte d'une discussion de la memoria, les actus humanae vitae. Boncompagno fait aussi montre d'une insistance nouvelle sur le caractère mémorial des images publiques, et affirme la liaison entre celui-ci et leur appartenance à un espace : peintures, sculptures, enseignes, mentionnées in situ, forment une procession de signes dont le lieu joue un rôle actif vis-à-vis de son objet ${ }^{21}$.

18. Cf. Marcia Kupfer, «The Lost Mappamundi at Chalavoy-Millon », Speculum, 66, 1991, pp. $540-571$.

19. Non sans rencontrer des opposants sceptiques. Jean de Salisbury déclare dans son Metalogicon, 1159, que l'art de la mémoire recommandé par Tullius ne lui paraît pas très utile. La Poetria nova de Geoffroy de Vinsauf estime la méthode des lieux et des images compliquée et ennuyeuse, et lui préfère celle des notulae, c'est-à-dire des notes ou marques dans la marge du livre.

20. Bologne et son école de droit, où l'enseignement de la rhétorique acquiert vite une grande importance, en est sans doute le point d'ancrage originel.

21. Vont dans le même sens une série de recommandations concernant les lieux d'études, dont certaines font étrangement écho aux recommandations hérenniennes. Boncompagno recommande d'y interdire les images étrangères aux sujets enseignés, pour éviter toute confusion, et d'y percer des fenêtres permettant au maître d'apercevoir la campagne, "puisque la mémoire trouve vigueur dans la vision d'objets agréables". Ainsi encadré, le paysage donne à 


\section{LECTURES DES GUVRES}

Si le domaine défini par Boncompagno pour l'efficace de la mémoire artificielle s'est démesurément agrandi - ainsi que dans une large mesure laïcisé - par rapport à un Hugues de Saint-Victor, le détail des techniques mnémoniques qu'il emploie est issu en droite ligne de la mémoire « hérenno-victorine» définie plus haut, et ne subit pas grand changement. Il est constitué, comme chez Hugues et ses successeurs, d'une série de diagrammes semés d'imagines agentes, dont certains devaient trouver place sur des picturae apposées aux murs des lieux d'études. De plus, si, de façon relativement neuve, la fabrication des monuments antiques est conçue par Boncompagno comme ayant eu un but explicitement mémorial, le livre reste pour lui, devant l'image, le meilleur instrument de la memoria 22 .

Il en va autrement des générations successives de dettatori et juristes, bolonais ou non, chez qui la problématique des lieux à la fois se répand et prend un tour résolument hérennien. Ce trait est sensible à la fois dans la diffusion de systèmes dont les lieux sont les pièces de maisons ou de palais ${ }^{23}$, dans la faveur extrême dont jouit la Rhétorique à Hérennius, couplée au De l'invention et auréolée du prestige accordé à Cicéron, «li mieus parlans hom del monde » selon Brunetto Latini ${ }^{24}$, et dans l'établissement neuf de volgarizzamenti de la Rhétorique. Ceux-ci, se multipliant dans la seconde moitié du siècle, permettent à un public non latinisant (illitteratus), d'avoir accès à ses techniques, soit passivement, soit aussi de manière active ${ }^{25}$.

La large diffusion d'une problématique hérennienne des lieux, au moins partiellement détachée du contexte monastique où elle avait repris force, a pour corollaire l'attention nouvelle portée à la lettre de ses règles, attention liée au rôle croissant de modèle que joue le passé romain antique, sous ses formes plastiques autant qu'écrites.

l'architecture du lieu ornement et variété, sans brouiller pour autant la production d'images de mémoire.

22. "Adrien, l'un des empereurs, en signe de souvenir éternel, fit sculpter de façon merveilleuse l'histoire de Troie (sic) sur une colonne, qu'il fit ériger à Rome. En effet, les Romains avec des sculptures, les Grecs avec des images de leurs hauts faits, voulurent que ceux qui les suivraient se souviennent d'eux. Mais les choses du passé se conservent avec des livres de façon plus valable qu'avec des sculptures et des images ", Boncompagno, Le siège d'Ancône.

23. Les prologues d'œuvres comme le Candélabre du Florentin Bene, la Gemme pourpre (1226-1227) de Guido da Faba, ou encore l'Instrument du droit civil d'Anselmo de Orto, en sont l'indice, qui mettent en scène des personnages "allégoriques » situés dans des lieux-supports des notions qui forment le sujet de l'œuvre. Anselmo décrit explicitement le «Temple de la Justice » qui sert de cadre à la scène comme une des maisons où il a vécu : "J'ai trouvé cette maison dans la ville de Bologne (...), et j'y ai longtemps résidé, et j'y ai tout visité attentivement, en enquêtant avec diligence ", Anselmo DE ORTO, Iuris Civilis Instrumentum, A. GaudEnZI éd., dans Bibliotheca iuridica Medii Aevi, II, 87.

24. B. LATINI, Le livre dou trésors.

25. Le plus célèbre d'entre eux, le Fiore di Rettorica composé entre 1258 et 1266 par Guidotto da Bologna, et réélaboré par Bono Giamboni, existe en plusieurs versions. Cf. F. TocCO, "Il Fior di rettorica e le sue principali redazioni secondo i codici fiorentini », dans Giornale storico della Letteratura italiana, XIV, 1902, pp.337-364. J'ai montré ailleurs comment ses trois versions principales dessinent différents publics de la mémoire: elles vont de l'absence complète de la section mnémonique de la Rhétorique, jugée trop complexe pour des illitterati, jusqu'à une traduction presque intégrale, qui permet son usage, en passant par un résumé qui en éclaire la pratique sans entrer dans le détail de ses règles. 
Elle a aussi pour conséquence la cassure définitive de la bipartition litterati/illitterati qui avait longtemps régi la hiérarchie du texte et de l'image et la conception de leurs publics.

\section{Publics et praticiens de l'image}

Durant le premier Moyen Age, la conception chrétienne de l'image s'était résolument située du côté de la tradition quintilianienne, maintenant un rapport strict de subordination de l'image au verbe ${ }^{26}$. Celui-ci prenait la forme soit d'une méfiance à l'égard de l'image, considérée comme supplément superflu ou dangereusement ambigu de l'écriture, soit de son assimilation à l'écriture, mais à une écriture d'ordre inférieur. C'est cette dernière position qui définit l'orthodoxie de l'Église depuis la lettre, vite fameuse, écrite en 600 par Grégoire le Grand à Serenus, évêque de Marseille, qui avait fait scandale en s'attaquant aux peintures qui ornaient ses églises. Grégoire, s'il y réfute l'idée que les images doivent être adorées, justifie leur légitimité en tant qu'elles sont le moyen d'instruire ceux qui n'ont pas accès à l'écriture ${ }^{27}$.

En définissant la perception de l'image "sur le mode de la lecture d'un texte écrit ${ }^{28}$, la lettre de Grégoire a longtemps servi à réitérer le contrôle de l'écriture sur l'image, et par là le contrôle des litterati, à même d'expliquer la seconde par la première, sur les illitterati. Mais elle a aussi eu pour effet de légitimer, à l'intérieur du cadre restreint du public des illitterati, l'équivalence structurelle image/texte revendiquée par la tradition hérennienne, et la logique qui la sous-tend, qui est celle de l'Ut pictura poesis.

On trouve trace de ce rapport différent à l'image dans une des trois lettres de Grégoire qui ont servi à définir la position de l'Église sur les images : la lettre à l'ermite Secundinus de mai 599. Celle-ci diffère de la première de deux manières : le passage concernant les images est apocryphe ${ }^{29}$; l'attitude envers les images qu'il définit est très différente de celle énoncée dans la lettre à Serenus. Selon l'interpolation, Secundinus ayant réclamé à Grégoire des images, celui-ci lui fait envoyer «deux panneaux peints qui contiennent l'image de Dieu sauveur et de sainte Marie mère de Dieu, et les bienheureux apôtres Pierre et Paul [...], et une croix ${ }^{30}$. Surtout, il approuve

26. Cf. Jean-Claude Schmitт, «Écriture et image : les avatars médiévaux du modèle grégorien ", dans Littérales. Théories et pratiques de l'écriture au Moyen Age, Paris, 1988, pp. 119-154, à qui j'emprunte une partie des considérations suivantes.

27. «Car c'est une chose d'adorer l'image, et c'en est une autre d'apprendre, par l'histoire montrée en peinture, ce qu'il faut adorer. En effet, ce que l'écriture est à ceux qui lisent, la peinture l'offre aux hommes incultes qui la regardent. C'est en elle que les ignorants voient ce qu'ils doivent suivre, en elle que lisent ceux qui ne connaissent pas les lettres. Pour ceux qui restent attachés au paganisme tout particulièrement, la peinture tient lieu de lecture. [...] Il ne faut donc pas briser [les images], car elles n'ont pas été placées dans les églises pour y être adorées, mais seulement pour servir à instruire les esprits de ceux qui ne savent pas ", GrÉGOIRE LE GRAND. Epistolae, dans Monumenta historica Germaniae, II, X, 10, Berlin, 1957, p. 270.

28. J.-Cl. Sснмiтt, art. cit., p. 123.

29. Il apparaît dans le texte de la lettre pour la première fois en 769 , au concile de Latran, sans doute donc en tant qu'élément du « débat sur les images qui, à cette époque déjà, semble avoir agité l’Église franque », Cf. J.-Cl. SCHмitr, art. cit., p. 125.

30. Grégoire le Grand, Epistolae, IX, 52, dans P. L. LXVII, col. 991b. 


\section{LECTURES DES CUVRES}

la demande de l'ermite en des termes qui définissent la destination de l'image par deux caractéristiques : sa liaison à l'affection et au souvenir, son équivalence directe avec l'écriture ${ }^{31}$. Elle n'a plus pour fonction de pallier l'inaccessibilité du texte ou de faire apprendre, mais est dirigée vers les affects du regardeur par la représentation qu'elle offre du souvenir.

S'il ne peut être question d'assimiler strictement la fonction mémoriale de l'image ici définie aux techniques de l'art de la mémoire, on ne peut que noter le terrain commun sur lequel elles se meuvent : la visualisation comme mise en présence d'un événement, la figuration indirecte, la prise en charge d'affects par le visuel, enfin le parallèle entre écriture et image, qui évoque la comparaison hérennienne entre lieux/tablettes et lettres/images. L'une et l'autre sont également capables de susciter la remémoration, au moyen de figures du souvenir qu'elles construisent chacune à leur façon.

C'est cette conception qui, restée en sommeil durant plusieurs siècles, a été réveillée par la renaissance monastique de la memoria au $\mathrm{XII}^{\mathrm{e}}$ siècle, pour former au Duecento et au Trecento une structure commune aux différents usages de l'image. Le renouveau de l'association hérennienne lieu/ image permet en effet la fécondation réciproque des images publiques didactiques, dont les fonctions et l'extension étaient longtemps demeurées restreintes, et des images individuelles de mémoire.

Cette nouvelle production d'images repose sur l'entrelacs entre image et parole, le visuel étant porteur de mots, soit par la narration qu'il donne à voir, soit encore par la présentation de jeux de mots incarnés dans l'image. Elle implique un degré souvent élevé de literacy, ainsi que de familiarité avec les objets présentés visuellement ${ }^{32}$. La ligne de partage entre litterati et illitterati, qui avait longtemps coïncidé avec celle qui séparait clercs et laïcs, se défait pour laisser place à une multiplicité de zones d'image définies chacune, à l'intérieur du cadre général de la memoria, par l'extension et la nature de leur public. Celles-ci dictent le type d'image utilisé, son degré de visualité et sa complexité ${ }^{33}$, du diagramme à l'historia, de la peinture infamante aux grands programmes théologico-politiques florentins ou siennois ${ }^{34}$.

La diffusion de l'art hérennien n'implique donc pas seulement l'apparition de nouveaux publics et praticiens de la memoria, mais celle de nouveaux publics et praticiens de l'image, qui mettent en branle l'essor extraordinaire pris par la production visuelle dans le courant du Duecento.

31. «Je sais pour sûr que tu ne recherches pas l'image de notre Sauveur afin de l'adorer, mais pour que par le souvenir du Fils de Dieu tu te réchauffes dans l'amour de celui dont tu désires voir l'image. Et nous aussi, nous nous prosternons devant elle non comme devant la divinité, mais nous adorons celui que son image, en nouveau-né ou mort ou siégeant sur son trône, nous rappelle. Et tandis que cette même image nous renvoie comme l'écriture au souvenir du Fils, notre esprit est réjoui par la Résurrection ou caressé par la Passion ", GréGoire LE Grand, ibid., coll. 990d-991a.

32. C'est en ce sens que l'on doit déclarer la plupart des images du second Moyen Age mémoriales plutôt que didactiques. Elles ne servent pas à apprendre quelque chose d'inconnu, mais à rappeler de façon efficace quelque chose de déjà connu, dont il s'agit de disposer de façon aisée. Cf. les remarques de Michael W. Evans, Medieval Drawings, Londres, 1969, p. 11.

33. Étant entendu que différents niveaux de lecture, des plus érudits aux plus « faibles ", subsistent pour une même image ou série d'images.

34. Cf. Jean-Philippe Antoine, art. cit., en particulier pp. 587-615. 
Cet essor est particulièrement remarquable dans le domaine de la sculpture et de la peinture murale, c'est-à-dire des images publiques, conçues de façon croissante comme des séries de scènes logées dans des architectures.

\section{De nouvelles images}

Si peintures, sculptures, et de façon générale éléments décoratifs n'ont jamais cessé d'être présents dans les églises, y compris jusqu'à Byzance durant les périodes iconoclastes, le $\mathrm{XII}^{\mathrm{e}}$ siècle et le $\mathrm{XIII}^{\mathrm{e}}$ siècle constituent dans l'Europe de l'Ouest une période d'activité artistique particulièrement intense, étroitement liée à la construction d'édifices ou à leur restauration. Cette activité est caractérisée par deux traits principaux: un renouveau sculptural fondé sur le remploi et l'étude de l'Antique ; et l'association de l'image (sculptée ou peinte) à un lieu.

Un premier moment de ce phénomène, proche de la mémoire victorine définie plus haut, est offert par la statuaire du XII ${ }^{\mathrm{e}}$ siècle, dont l'ordonnance dans l'architecture ecclésiale est la marque d'une problématique neuve de la mise en place d'images dans l'espace physique du regardeur. On rapprochera par exemple les séries linéaires de personnages bibliques et historiques que déploie l'image hugonienne de l'Arche, ou encore celles qui ornent le Tabernacle d'Adam le Prémontré, des statues-colonnes de la cathédrale de Chartres $^{35}$. Mais au-delà de cette première ressemblance d'arrangement, déjà suggérée autrefois par F. Yates, les problèmes plastiques et spatiaux qu'incarne cette statuaire commandent un rapprochement plus concret. Comme l'a montré, partiellement à la suite de E. Panofsky, Louis Grodecki ${ }^{36}$, les statues gothiques se distinguent de leurs précédents romans par le rapport neuf qu'elles entretiennent avec l'architecture où elles prennent place. Là où la statuaire romane est directement subordonnée à la fonction de l'architecture qui la supporte et demeure à l'intérieur d'un "plan mural » qui en limite le volume ${ }^{37}$, les développements gothiques se caractérisent par ce que L. Grodecki nomme la « règle de l'adossement ». D'une part, la statue se détache du mur ou de la colonne dont elle faisait jusque-là partie : elle est le plus souvent sculptée indépendamment d'eux, et acquiert un volume autonome. D'autre part, elle devient étroitement associée à un site que délimitent un socle, et aussi souvent un dais ou des arcatures, sans oublier le mur auquel elle est adossée comme devant un fond ${ }^{38}$. L'ensemble forme une

35. Cela implique que les pratiques de l'art de la mémoire constituent le modus operandi concret qui relie scolastique et architecture gothique, modus operandi parfois poursuivi par E. Panofsky, à son corps défendant, sous forme d'un simple parallélisme ou d'une relation abstraite. Cf. E. PANOFSKY, Architecture gothique et pensée scolastique, Paris, 1967.

36. Louis Grodecki, "Problèmes de l'espace dans la définition de la sculpture gothique ", dans Le Moyen Age retrouvé, Paris, 1991, t. II, pp. 109-117.

37. «La forme sculptée [...] est claveau d'archivolte, angle de chapiteau, côté de trumeau, portion de tympan. Elle fait partie du mur matériellement, par l'engagement de ses pierres dans les assises de la bâtisse ; optiquement, parce que sa face extérieure ne franchit pas le plan du mur, elle n'en est qu'une ondulation, un accent... », ibid., p. 112.

38. A cette caractéristique de la statuaire correspond dans le registre des hauts et bas-reliefs l'importance nouvelle donnée au cadre, que l'on retrouve également dans la miniature. 


\section{LECTURES DES GUVRES}

niche spatiale dont la raison d'être est de faire exister le lieu auquel est associée la figure ou la scène représentée, lieu qui la distingue de ses voisines.

Cette présentation du lieu, pour la première fois depuis l'Antiquité romaine, sous forme d'une spatialité habitable, et non plus seulement, comme dans la peinture ou la sculpture romane, sous celle d'un signe disposé sur une surface, a pour corollaire l'évolution des figures sculptées. Ici encore, sous l'influence neuve de modèles antiques, celles-ci acquièrent une prétention neuve à la ressemblance du vivant et de ses gestes. L'un et l'autre développements sont l'indice d'une problématique hérennisante, où des êtres non visuels et des énoncés prennent figure sous forme d'imagines frappantes et vives, qui habitent une série de lieux spatialement ordonnés.

La même problématique informe les développements picturaux qui prendront bientôt place de l'autre côté des Alpes, mais elle y produit des effets originaux, grâce à la présence d'une tradition spécifique : celle de la peinture murale romaine et paléo-chrétienne ${ }^{39}$, déjà objet de "renaissances " périodiques $^{40}$, à laquelle elle va donner une actualité nouvelle.

La présence dans les développements italiens du Duecento et du Trecento d'éléments empruntés à la peinture romaine antique est tout aussi impossible à nier que difficile à documenter autrement que par des comparaisons formelles. Une des difficultés du débat gît dans l'approche habituellement adoptée, qui procède par l'identification d'emprunts de détail plutôt que par celle de problématiques structurelles communes ${ }^{41}$. L'hypothèse d'un contact direct de peintres comme Cavallini ou Giotto avec des monuments peints antiques depuis disparus, dans la mesure où elle ne peut trouver de caution documentaire écrite, doit alors affronter la question suivante : si ces monuments étaient visibles et disponibles depuis des siècles, pourquoi n'ontils pas pénétré plus tôt la pratique artistique ? Le lien neuf entre memoria et pratique hérennienne des images permet de formuler une possible réponse à cette question, sous forme d'une double hypothèse.

La problématique de l'image développée par la diffusion de la culture hérennisante de la memoria rend de nouveau visible et interprétable pour les peintres et leurs commanditaires la peinture murale antique, ou plutôt ses monuments, dont les caractéristiques spatiales avaient depuis longtemps perdu leur intelligibilité. Ceux-ci, à tort ou à raison ${ }^{42}$, sont en effet à la fois perçus comme possédant une destination mémoriale et associés au traite-

39. Les $\mathrm{XIII}^{\mathrm{e}}$ et $\mathrm{XIV}^{\mathrm{e}}$ siècles sont loin de distinguer l'une de l'autre.

40. Cf. Hélène Toubert, "Le renouveau paléo-chrétien à Rome au début du XII siècle ", Cahiers archéologiques, 1970, pp.99-154. Sur le statut des différents styles romains dans ces « renaissances ", voir aussi I. LAviN, « The Ceiling Frescoes in Trier and Illusionism in Constantine Painting », Dumbarton Oaks Papers, 21, 1967.

41. Cf., outre les articles cités dans la note précédente, Mariette DE Vos, «La ricezione della pittura antica fino alla scoperta di Ercolano e Pompei ", dans Memoria dell'Antico nell'arte italiana. II. I generi e i temi ritrovati, Turin, 1985, pp. 351-380. On opposera à ses conclusions plutôt négatives les travaux de John White, tout comme ceux de A. SMART, The Assisi Problem and the Art of Giotto, Londres, 1971, et Hans Belting, Die Oberkirche von San Francisco in Assisi : ihre Dekoration als Aufgabe und die Genese einer neuen Wandmalerei, Berlin, 1977.

42. Sans doute à raison. Voir à ce sujet Agnès Rouveret, Histoire et imaginaire de la peinture ancienne, Rome, 1989, et la communication citée plus haut. Voir aussi JeanPhilippe Antoine, «L'arte della memoria e la trasformazione dello spazio pittorico », dans $L a$ cultura della memoria, cit. supra. 
ment hérennien de l'art de la mémoire, qui leur restitue une signification globale.

L'association des monuments peints antiques à la memoria, liée à la situation propre de l'Italie, et en particulier de Rome, où ceux-ci étaient plus nombreux que partout ailleurs, donne sa direction spécifique à la « révolution picturale » du Duecento, révolution qui reste longtemps locale, malgré l'extension européenne des pratiques de la memoria. Les fragments de peinture romaine ou paléo-chrétienne, dotés par la memoria d'une intelligibilité nouvelle, vont fournir des modèles proprement picturaux de figuration spatiale des lieux, et permettre l'avènement d'un type neuf de spatialité dans la peinture.

Ce travail a pour premier moment la question de la mise en place de l'image. En témoigne la faveur nouvelle dont jouit un système de présentation qui, sans avoir jamais complètement disparu, au moins dans la peinture murale de la péninsule italienne, n'avait plus qu'une importance limitée: celui des bandes narratives, chacune constituée de rectangles contenant une scène. En témoigne aussi le fait que la résurgence de formules décoratives romaines ou romanisantes s'effectue d'abord à l'extérieur des scènes, dans le décor qui leur sert de cadre et définit leur lieu : le mur, divisé en bandes étagées, les bordures des scènes, formées de colonnes et chapiteaux, qui reproduisent parfois ceux de l'architecture du lieu. La production picturale d'un lieu spatialement défini est donc d'abord celle d'un lieu pour l'image. Elle établit, ou plutôt rétablit, un lien entre la spatialité physique de la région qui abrite les peintures et les images que celles-ci donnent à voir.

Mais la question de la production picturale du lieu émigre bientôt du lieu extérieur de la scène picturale à l'intérieur de celle-ci. Ce n'est plus seulement l'historia qui réclame d'être située et ordonnée spatialement, mais les différentes imagines qui la composent.

\section{Le travail des lieux de Cavallini à Giotto}

La restauration d'un cycle de scènes de l'Ancien Testament à Saint-Paul hors-les-murs à Rome, confiée à Cavallini et à ses collaborateurs dans les années 1280 , fournit un des premiers et des plus puissants exemples de cette évolution ${ }^{43}$. La coexistence accidentelle de la version primitive et de la version « restaurée » d'une des scènes - le Massacre des innocents ${ }^{44}$ — permet de mesurer l'apport spécifique de l'atelier de Cavallini et la direction dans laquelle s'effectue son travail (fig. 1).

43. L'incendie de la basilique en 1823 en a rendu impossible en les détruisant l'analyse directe, mais une série de copies à l'aquarelle exécutées en 1634 pour le cardinal Barberini permet de connaître leur composition. Cf. S. WAETzold, Die Kopien des 17 Jahrhunderts nach Mosaiken und Wandmalereien in Rom, Vienne, 1964. Voir aussi John White, "Cavallini and the Lost Frescoes in San Paolo ", Journal of the Warburg and Courtauld Institutes, XIX, 1956, et Paul Hetherington, Pietro Cavallini, Londres, 1979.

44. La réduction effectuée par les restaurateurs du programme original des peintures, de quarante-deux à trente-sept scènes, et l'inachèvement probable de l'entreprise ont conduit à laisser subsister, au côté de la scène "restaurée ", la peinture d'origine. 


\section{LECTURES DES GUVRES}

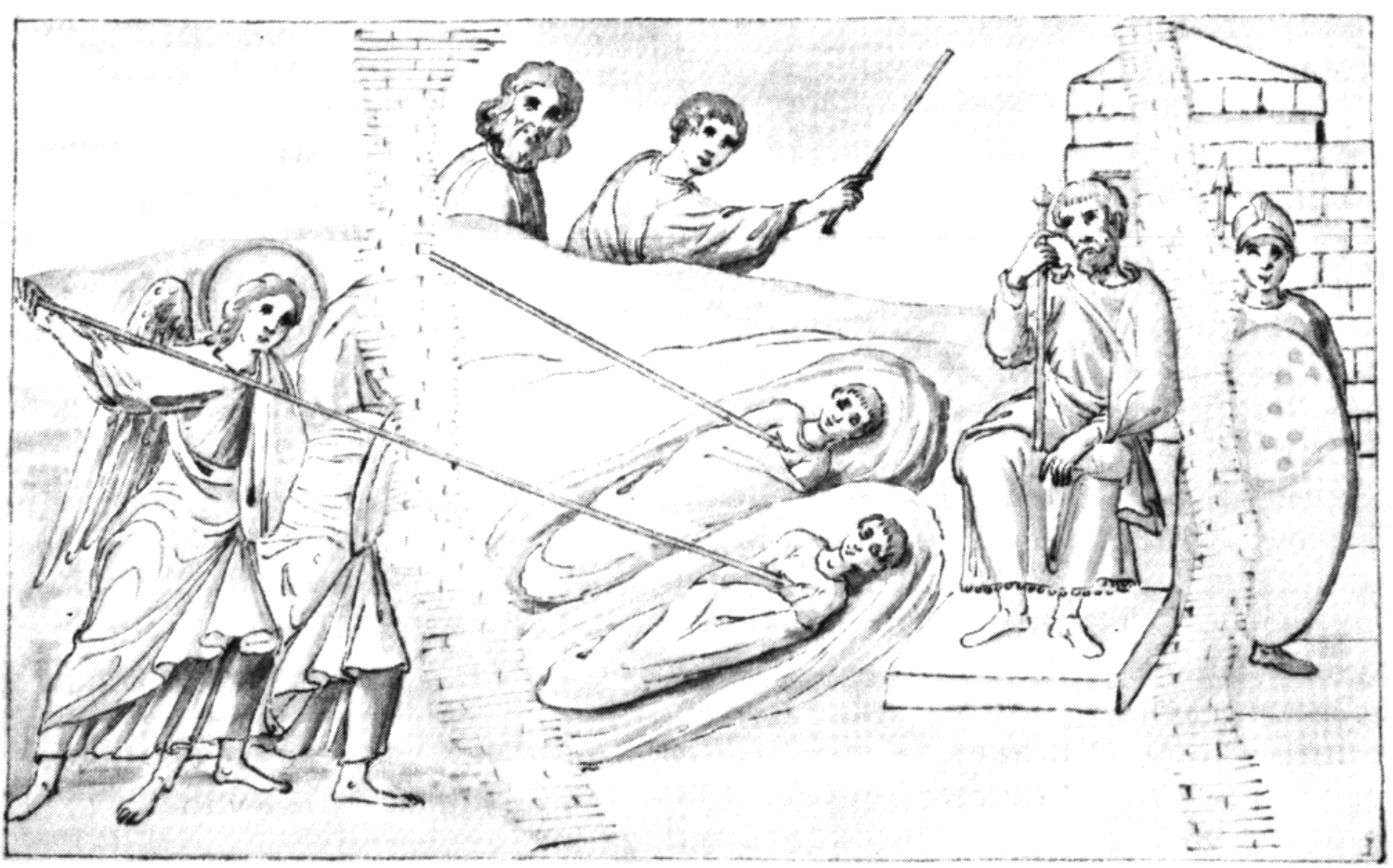

FIG. 1. -- Massacre des Innocents, d'après un anonyme (ci-dessus) ; d'après Pietro Cavallini (ci-dessous). Saint-Paul-hors-les-murs, Rome, Ms. Vat. Barb. Lat. 4406, f. $62 \mathrm{r}$.

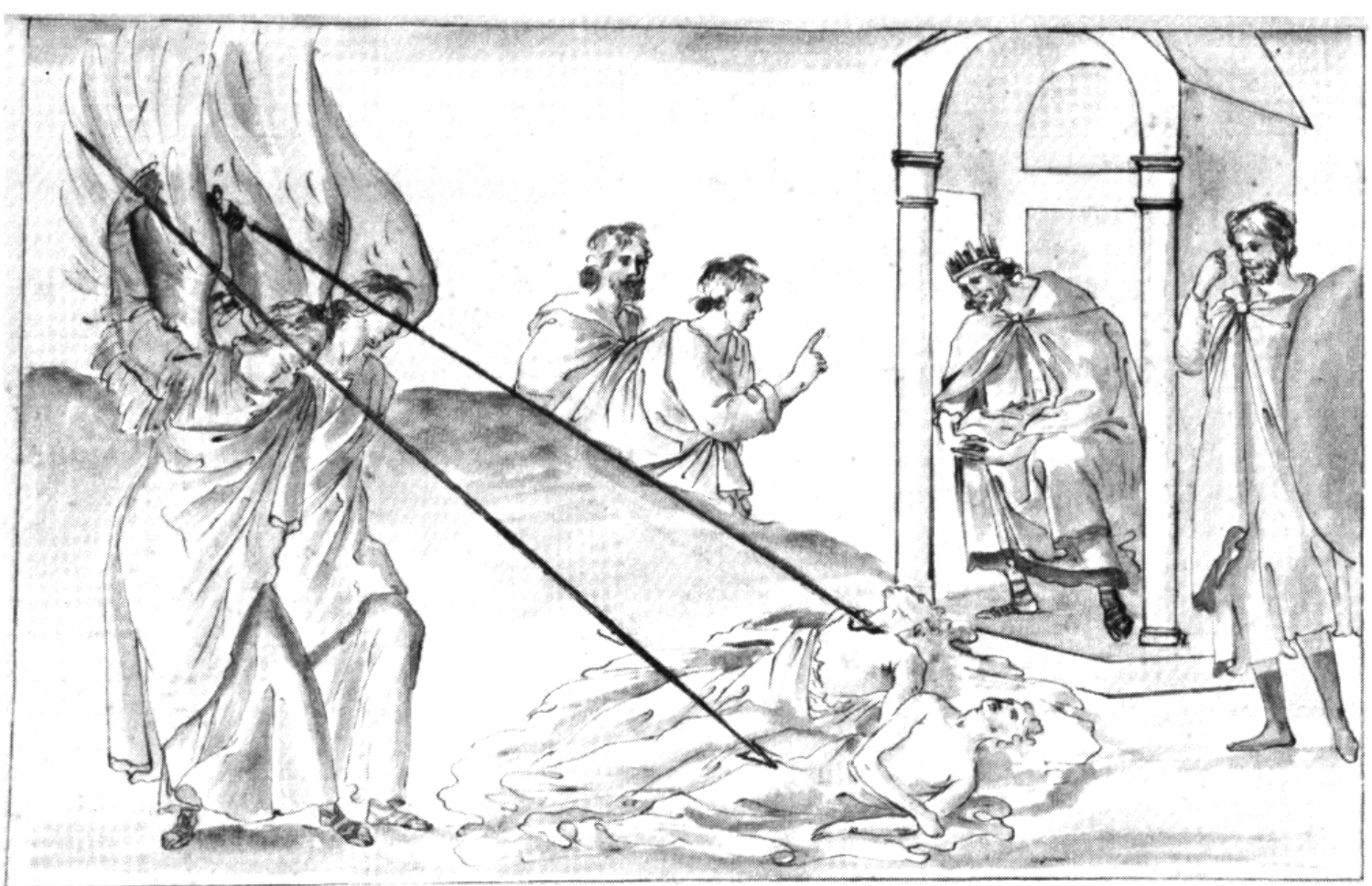




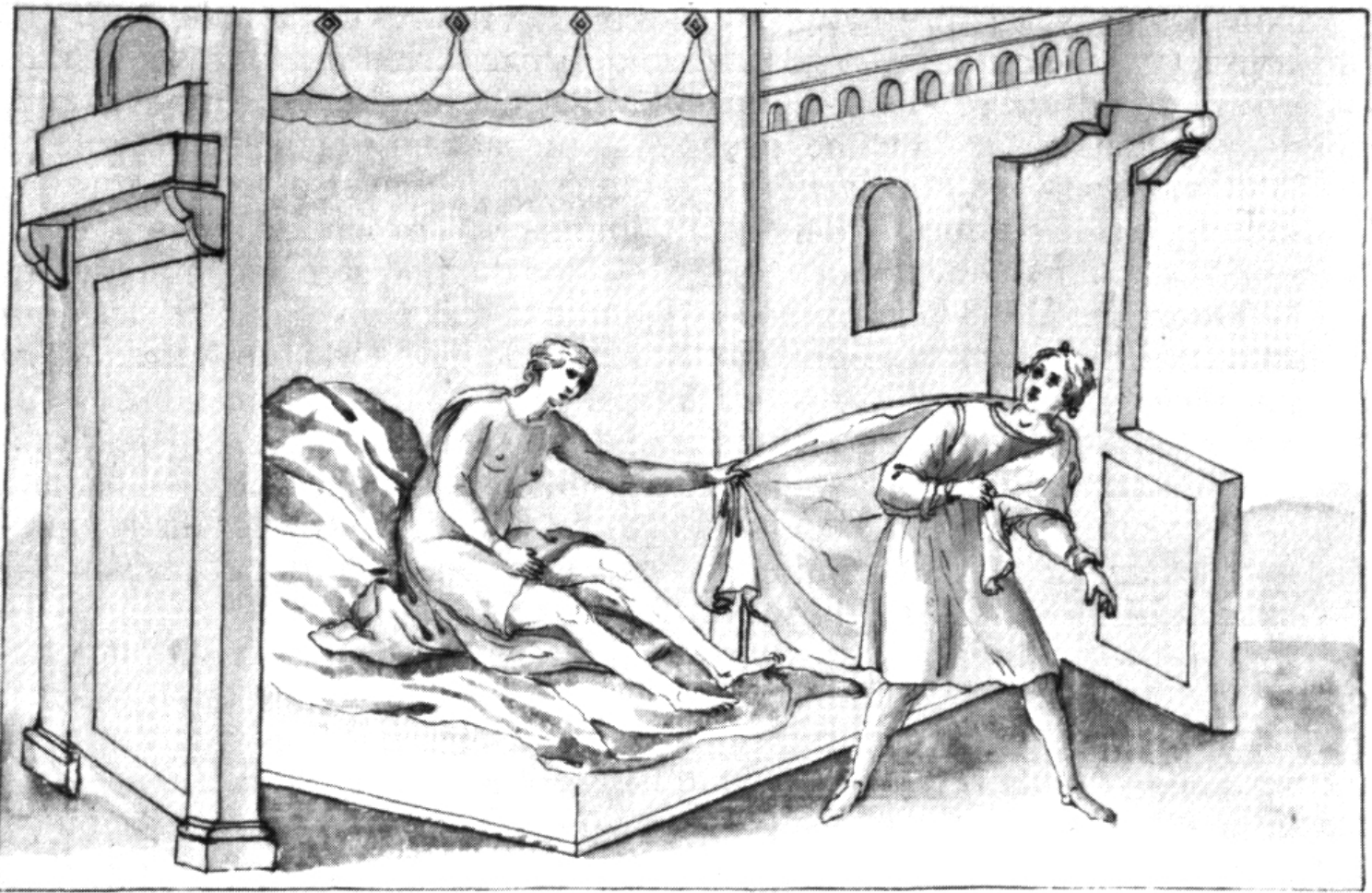

Fig. 2. - Pietro Cavallini (d'après), Joseph et la femme de Putiphar (ci-dessus), Joseph en prison (ci-dessous), Saint-Paul-hors-les-murs, Rome, Ms. Vat. Barb. Lat. 4406 , f. 62 r.

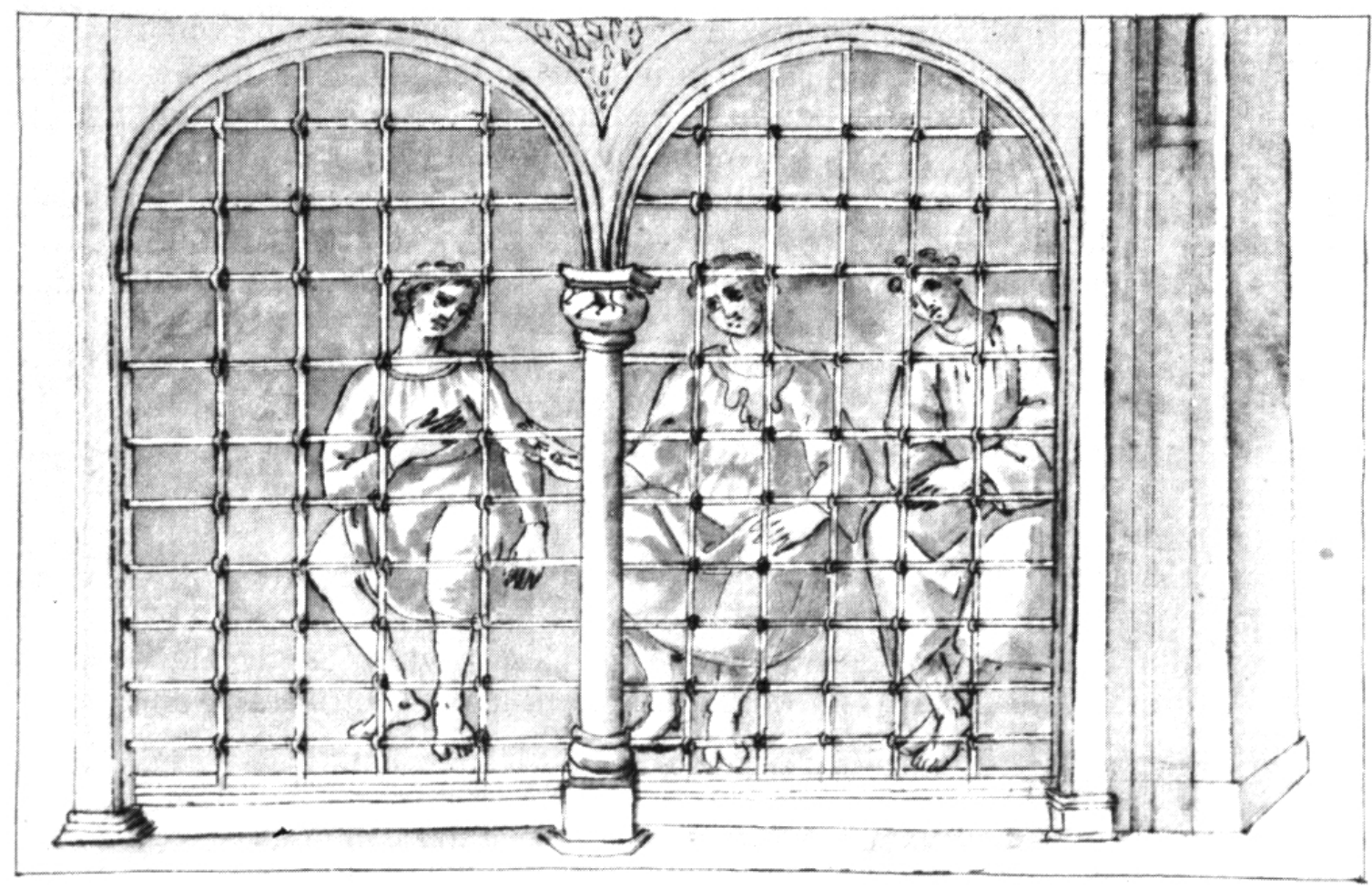




\section{LECTURES DES GUVRES}

Dans les deux cas, l'implantation globale des figures est la même : Cavallini conserve donc autant que possible le dessin antérieur de la fresque. Mais sa version en diffère par la mise en espace des figures. Pharaon, dans la première version assis sur un trône, occupe maintenant un édicule représenté en profondeur. C'est de l'intérieur de celui-ci qu'il converse avec Moïse et Aaron, conversation que rendait impossible dans la première version le placement de la colline, qui isolait Moïse et Aaron du massacre accompli par les anges. De même, dans la première version de la scène du massacre, les nouveau-nés dorment dans des berceaux en forme de mandorle qui flottent dans le plan pictural entre les anges et Pharaon. Dans l'image "restaurée ", ils agonisent dans leurs langes défaits, sans que rien les sépare du reste du champ pictural ${ }^{45}$. Enfin, là où la version originelle donne même dimension à toutes les figures, la taille des personnages cavalliniens sert à les distribuer à distance les uns des autres, diminuant des anges vers Pharaon, et plaçant par exemple celui-ci au fond de la scène.

Au total, Cavallini, tout en conservant la division diagonale du plan pictural que forme la colline, dote ce plan d'une signification neuve. L'ensemble des éléments présents, architecture et figures, se conjugue pour former une véritable région spatiale, qui enferme les deux lieux du massacre et de la conversation. C'est la structure lieu/image qui règle maintenant l'organisation picturale de l'historia.

L'examen de l'ensemble du cycle renforce les conclusions de l'examen comparé du Massacre des premiers-nés. Celui-ci témoigne d'un même travail de construction du plan pictural en région-pour-des-lieux, construction réalisée à la fois par l'insertion des figures dans une architecture, et par de véritables effets de profondeur: dans Joseph et la femme de Putiphar, par exemple (fig. 2), les deux personnages sont enclos à l'intérieur d'une pièce que définissent trois murs, dont deux latéraux vus en récession, l'un de l'extérieur, l'autre sous forme de paroi intérieure, et le lit, lui aussi présenté comme un volume. Un autre exemple est celui de Joseph en prison (fig. 2), scène dans laquelle la grille qui ferme la prison, placée entre le regardeur et les figures des prisonniers, augmentée de la récession du mur latéral droit, les situe clairement à l'intérieur d'une pièce dont ils occupent le volume. Les précédents d'une telle construction sont tous bas-antiques, et renvoient à des modules de construction plus anciens.

Le travail de Cavallini sur les peintures existantes consiste donc à faire exister dans l'espace même de l'historia la structure lieu/image qui sert de cadre à l'ensemble du programme des fresques. De même que chaque scène a son lieu dans l'architecture du bâtiment qui abrite leur série, de même, à l'intérieur de chacune, les imagines se distinguent les unes des autres, ou agissent les unes sur les autres en fonction des lieux définis qu'elles occupent.

Les vingt à trente années qui séparent les travaux cavalliniens de la maturité giottesque voient le développement et l'approfondissement de

45. Il est inutile d'insister sur la différence de valeur expressive de l'image, la seconde plus frappante et sanglante que la première, imago véritablement agens. 
cette logique. D'une part, la construction du lieu comme "boîte locale " ${ }^{46}$ à l'intérieur du champ pictural trouve une expression de plus en plus rigoureuse ; d'autre part, cette rigueur nouvelle permet la multiplication, à l'intérieur de ce même champ, de lieux distincts ainsi définis.

Un des premiers exemples de cette nouvellé construction du lieu dans le champ pictural appartient à la décoration de la basilique supérieure d'Assise, qui fournit comme un microcosme des transformations en cours dans la peinture italienne au tournant du siècle. Il s'agit de deux scènes de l'histoire d'Isaac, Jacob reçoit le droit d'aînesse (fig. 3) et Isaac repousse Esaü.

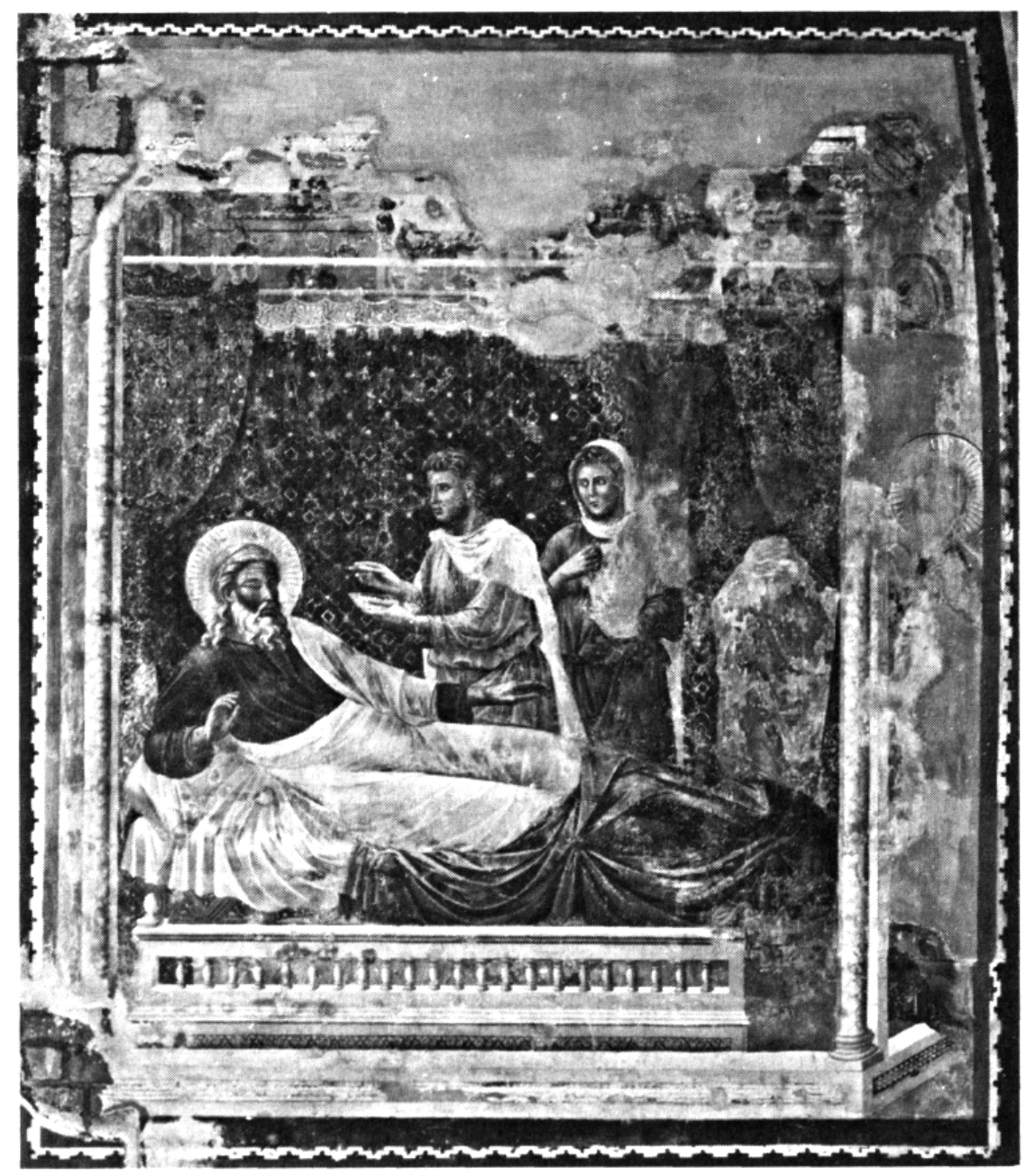

FIG. 3. - «Maitre d'Isaac», Jacob reçoit le droit d'aînesse, Église supérieure de Saint-François, Assise, Éd. Alinari, Pe 2a, no 5246.

46. En adoptant l'expression de «boîte locale », je fais ici référence à l'expression panofskienne de «boîte d'espace », mais détachée des connotations perspectivistes et néo-kantiennes qu'implique chez lui le concept d'espace. Comme on va le voir, la boîte locale fait exister une scène tridimensionnelle limitée; elle n'est pas un fragment d'un espace infini, choisi par le regard du peintre, et n'implique pas de point de vue unifié mathématiquement. 


\section{LECTURES DES GUVRES}

Autrefois attribuées au jeune Giotto, ces deux scènes le sont aujourd'hui plus communément à un peintre d'école romaine vraisemblablement formé par Cavallini : le "Maître d'Isaac». Elles sont caractérisées par le fait qu'elles prennent place dans une même «boîte locale ", qui envahit la presque totalité du champ pictural : la chambre d'Isaac, figurée sous forme d'une loggia à colonnettes typique de l'architecture romaine contemporaine. L'angle sous lequel sont figurés les murs latéraux les fait recéder vers la droite, et donne au plan frontal une qualité d'écran transparent, derrière lequel se trouve le lit sur lequel est étendu Isaac, lui-même représenté en récession, presque parallèlement au mur latéral. Le caractère sculptural des figures, qui renvoie à la sculpture antique, le jeu de la lumière sur leur visage et leur vêtement, en augmentant leur relief, accentuent l'effet de profondeur.

En même temps - et ceci n'est pas moins important - les effets de profondeur n'ont pas pour objet d'illimiter un espace. La spatialisation est au contraire limitée, à la fois par l'étroitesse des murs latéraux de la loggia, et par la tenture qui, placée de part et d'autre du lit, restreint l'espace de la scène à une alcôve. La tridimensionalisation du champ pictural s'estompe derrière les protagonistes. Tout se passe comme si, une fois créé un lieu pour l'action des figures, celle-ci perdait sa nécessité.

C'est ce que montre aussi la position de la «boîte locale » dans le champ pictural : la loggia où prend place la scène, si elle occupe la presque totalité du champ défini par les bandes horizontales et verticales qui séparent les différents panneaux, est distincte de celui-ci. Elle permet de définir un lieu spatial distinct du lieu comme simple place dans la série narrative ${ }^{47}$. C'est ce lieu spatialement délimité que les peintres vont apprendre à multiplier dans le champ pictural d'un même panneau, comme en témoigne la maturation progressive du travail de Giotto.

A Padoue, dans les fresques de la chapelle Scrovegni, les boîtes locales n'occupent plus la presque totalité du champ pictural, mais par leur présence elles le transforment en une région-pour-les-lieux elle-même virtuellement tridimensionnelle, manifestée par le bleu générique qui sert de fond. A l'intérieur de celle-ci, des architectures, la plupart fragmentaires, servent soit, comme dans la Pentecôte (fig. 4), à abriter l'ensemble d'une scène, soit, plus souvent, comme dans l'Annonciation (fig. 4) ou la Présentation de la Vierge au temple (fig. 4), à marquer, et ainsi à les différencier, les protagonistes de l'action. Dans le premier cas, un lieu à la profondeur spatiale limitée est défini par la coque de l'architecture, figurée en récession. Dans le second, les éléments architecturaux, eux aussi vus en récession mais du dehors, servent à tridimensionaliser le champ pictural. La dialectique des pleins et des vides permet alors de définir une variété de lieux sur une même scène.

Cette logique est développée à plein dans les fresques plus tardives exécutées par Giotto à Florence, dans la chapelle Peruzzi de Santa Croce. Le

47. Le procédé permet également de définir un second lieu pictural «négatif » dans le champ : à l'extérieur de la loggia, est peinte la figure, aujourd'hui presque entièrement effacée, d'un Jacob littéralement expulsé de la construction peinte à gauche. C'est le procédé qu'utilisera bientôt Giotto à Padoue dans Joachim chassé du temple. 
peintre y juxtapose dans le champ pictural des unités architecturales dont chacune enclôt une partie des protagonistes des scènes représentées. Cette multiplication des boîtes locales permet d'augmenter le nombre des personnages dans une scène sans faire foule, chaque lieu étant rarement occupé par plus de trois à quatre imagines. Elle permet aussi de dilater la spatialité du champ pictural, c'est-à-dire de la région-pour-les-lieux, avec pour résultat d'accentuer le caractère frappant et la présence visuelle des scènes figurées. C'est ce que montre par exemple la comparaison de la maison de la Naissance de saint Jean Baptiste florentine (fig. 5) avec celle de l'Apparition à sainte Anne padouane (fig. 6).

De la chapelle Scrovegni aux derniers travaux florentins, l'accomplissement giottesque réside, d'une part, dans la construction, à l'aide des boîtes locales partiellement héritées de la peinture antique et de la problématique mémoriale qui leur est associée, de lieux définis en termes de spatialité architecturale ; d'autre part, dans la dilatation de la région-pour-les-lieux (le champ pictural, doté de spaciosité par les boîtes qui l'occupent) à partir de la définition d'un point de vue visuel qui, quoique globalement homogène, n'est pas identifié à l'œil d'un spectateur immobile. Cette homogénéité du champ pictural conçu comme région-pour-les-lieux aboutit à définir, plutôt qu'un point de vue unique, une zone de compatibilité des différents effets de profondeur, à l'intérieur de laquelle le regardeur peut déambuler, sans que ses déplacements les annulent, et qui le fait dans une certaine mesure passer d'un lieu à un autre dans le champ pictural. Les effets de profondeur restent ainsi globalement compatibles, bien que jamais ajustés selon les règles d'une perspective exacte.

Plutôt que de voir dans cette évolution le jet encore hésitant d'un trait qui aboutit un siècle plus tard à la perspectiva artificialis et au tableau conçu comme fenêtre, on s'interrogera alors sur les limites qu'assignent à leurs accomplissements "spacieux » et Giotto lui-même et les peintres qui lui succèdent: comment par exemple les coretti de la chapelle Scrovegni, ces "fenêtres" qui ouvrent sur deux chambres imaginaires de chaque côté du chœur, et qui ont souvent été saluées comme la première authentique " perspective " dans la peinture occidentale, peuvent-ils coexister, dans le cadre d'un projet cohérent, avec les effets de profondeur "anti-perspectivistes " des loges de l'Annonciation qui les surplombent (fig. 4) ? Comment se fait-il qu'aucun peintre n'ait, pendant presque un siècle, poussé beaucoup plus loin l'homogénéisation "spacieuse » du champ pictural ? Pourquoi des effets de profondeur poussés ne s'accompagnent-ils pas d'une volonté de cohérence de l'espace ? Ces questions, insolubles si l'on se réfère à la vulgate perspecti$v^{\text {viste }}{ }^{48}$, trouvent un commencement de réponse si on considère la spaciosité

48. Elles réclament plus que la réponse alternative qui s'est développée depuis la perte de terrain de l'idéologie perspectiviste : celle de nécessités narratives qui entrent en conflit avec la volonté de cohérence pour l'espace. Si l'on a bien affaire, dans les ensembles de fresques jusqu'ici considérés, à un problème de structure narrative (y compris dans des peintures dont le contenu de sens est loin d'être immédiatement narratif), ce fait à lui seul n'autorise aucune conclusion, tant que la structure mémoriale de cette narration, et les pratiques qu'elle met en jeu, n'ont pas été mises au jour. 


\section{LECTURES DES GUVRES}
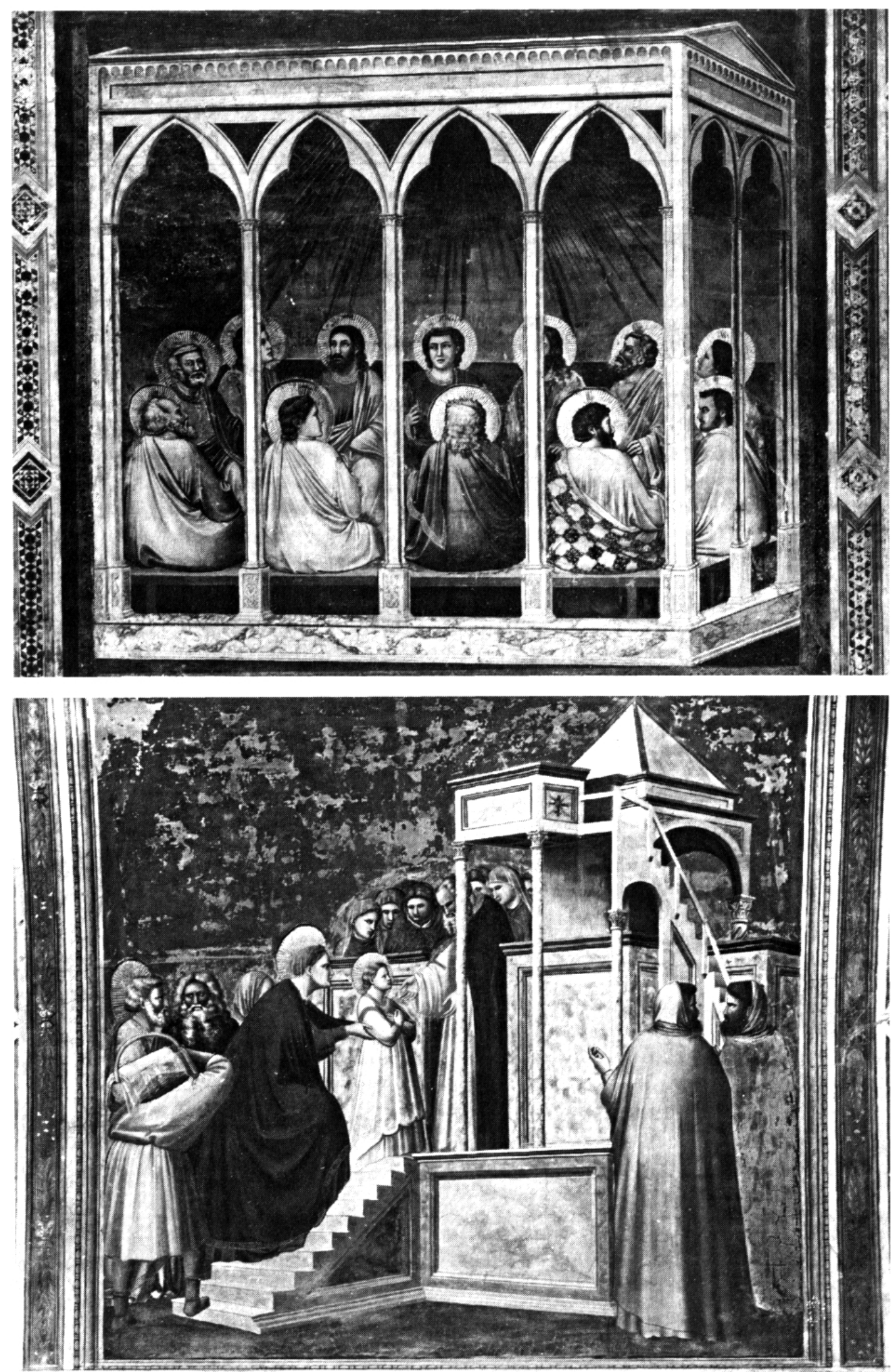


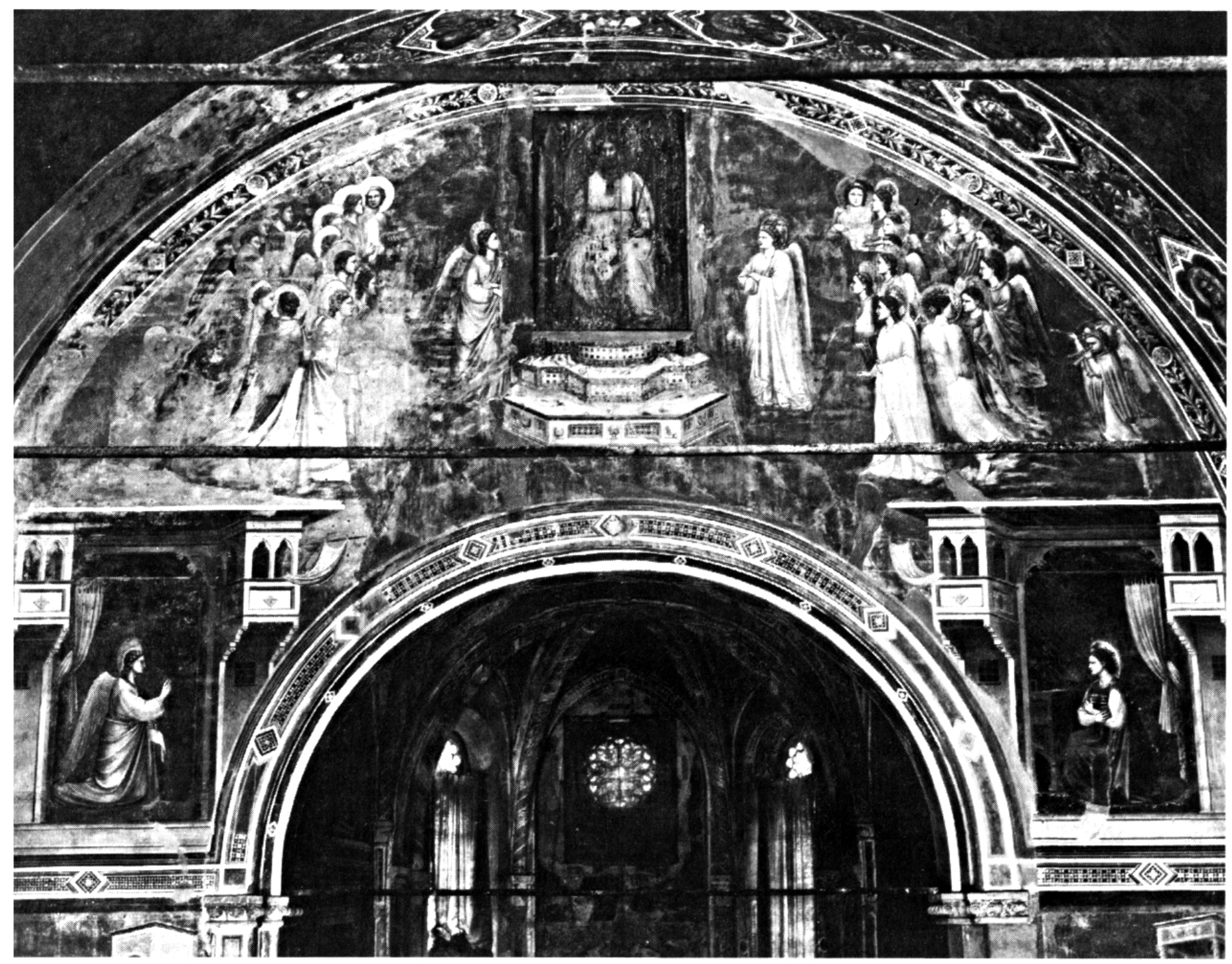

FIG. 4. - Giotto, Chapelle Scrovegni all'Arena, Padoue, Annonciation, Éd. Alinari, no 19416 (ci-dessus),

Dentecôte, Éd. Alinari, nº 19374 (ci-contre en haut),

Drésentation de la Vierge au temple, Éd. Alinari, nº 19316 (ci-contre en bas).

hétérogène nouvellement déployée par les peintres comme l'indice de la logique mémoriale de construction de lieux à l'œuvre dans la peinture : la spatialisation des lieux en est une exigence, comme leur pluralité et leur variété.

En clarifiant les liens qui unissent la memoria hérennisante et les problématiques d'invention spatiale qui transforment la peinture du Duecento et du Trecento, on n'a pas cherché à substituer à d'autres une nouvelle clef unique d'interprétation. La mise en rapport des pratiques d'invention mnémonique de lieux avec celles de l'invention picturale est loin de supprimer ou de rendre impertinentes les recherches iconographiques locales, ou encore les questions stylistiques et historiques touchant aux œuvres singu- 


\section{LECTURES DES GUVRES}

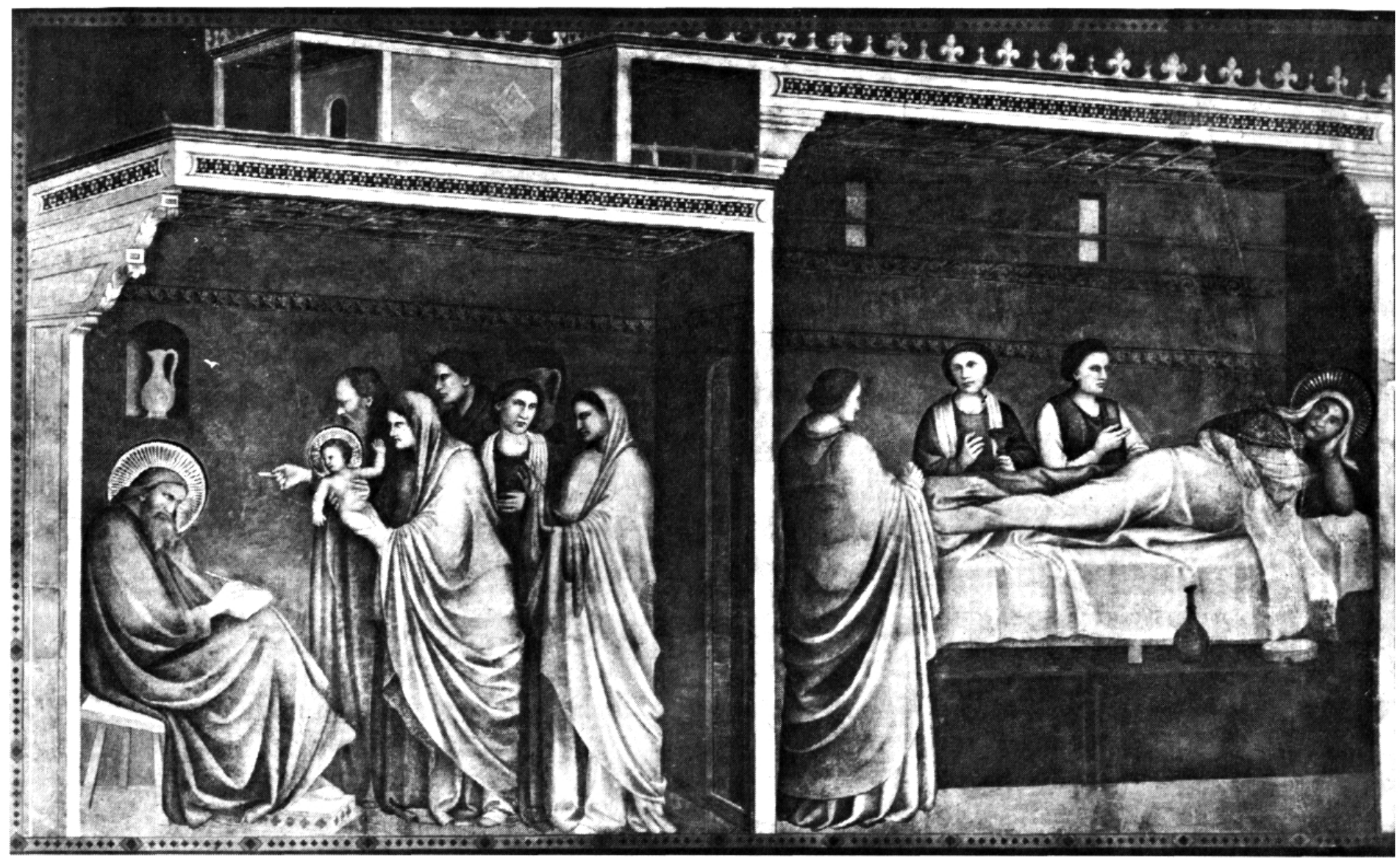

FIG. 5. - Giotto, Naissance de saint Jean Baptiste, Église de Santa Croce, Florence, Éd. Alinari, n 3929

lières. Mais elle a le mérite de les asseoir sur une fondation plus solide. Tout d'abord, elle permet de casser la construction perspectiviste qui sous-tend, le plus souvent de façon impensée, l'examen stylistique de la peinture des Duecento et Trecento, et avec elle la téléologie implicite qui y voit les balbutiements contradictoirement infantiles et géniaux d'un processus dont la vérité appartient à la Grande Renaissance. La distance de plus d'un siècle qui sépare les réalisations spatiales les plus audacieuses de Giotto de l'invention brunelleschienne de la perspectiva artificialis et de sa théorisation par Alberti, la coexistence, parfois dans une même historia, de constructions hétérogènes d'effets de profondeur, cessent alors de représenter un retard et une impuissance technique dans la réalisation d'un "espace de la peinture " identifié à l'espace perspectif. Ils appartiennent à un système différent, dont le but est de construire, dans la logique des pratiques mémoriales, des séries 


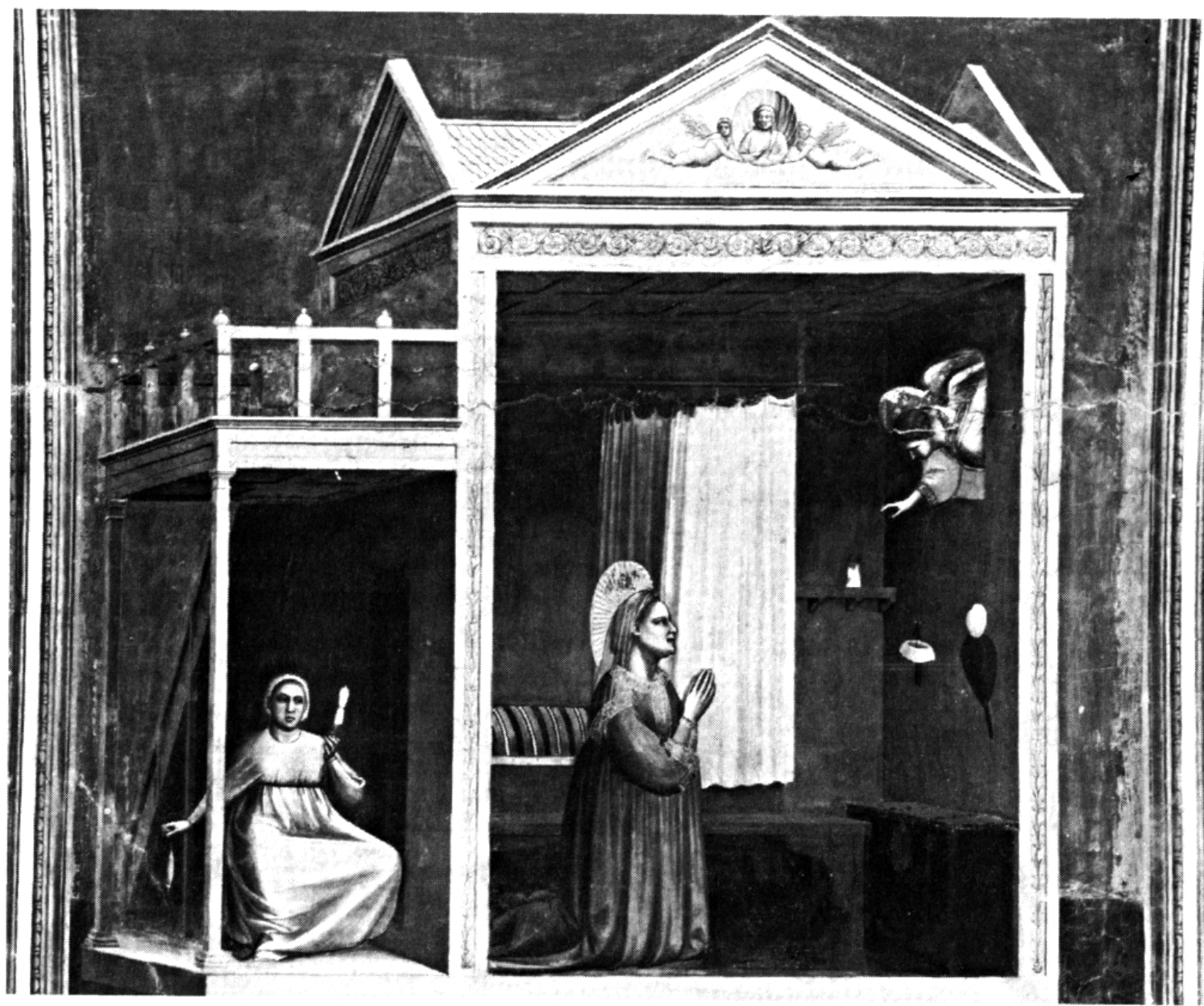

FIG. 6. - Giotto, Apparition à sainte Anne, Chapelle Scrovegni all'Arena, Padoue, Éd. Alinari, nº 19310.

de lieux peints spatialement définis, à l'intérieur d'une région picturale ellemême liée à son environnement architectural, et de recréer, à l'échelle du regardeur, les images qui peuplent son esprit, contribuant en retour à en fixer la forme.

Jean-Philippe AntoIne

Université de Lyon III 